\title{
Genome scale analysis of pathogenic variants targetable for single base editing
}

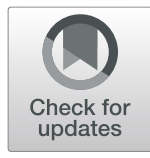

\author{
Alexander V. Lavrov ${ }^{* *}$ (D), Georgi G. Varenikov² and Mikhail Yu Skoblov ${ }^{1,2,3}$ \\ From 11th International Young Scientists School "Systems Biology and Bioinformatics" - SBB-2019 \\ Novosibirsk, Russia. 24-28 June 2019
}

\begin{abstract}
Background: Single nucleotide variants account for approximately $90 \%$ of all known pathogenic variants responsible for human diseases. Recently discovered CRISPR/Cas9 base editors can correct individual nucleotides without cutting DNA and inducing double-stranded breaks. We aimed to find all possible pathogenic variants which can be efficiently targeted by any of the currently described base editors and to present them for further selection and development of targeted therapies.

Methods: ClinVar database (GRCh37_clinvar_20171203) was used to search and select mutations available for current single-base editing systems. We included only pathogenic and likely pathogenic variants for further analysis. For every potentially editable mutation we checked the presence of PAM. If a PAM was found, we analyzed the sequence to find possibility to edit only one nucleotide without changing neighboring nucleotides. The code of the script to search Clinvar database and to analyze the sequences was written in R and is available in the appendix.

Results: We analyzed 21 editing system currently reported in 9 publications. Every system has different working characteristics such as the editing window and PAM sequence. C > T base editors can precisely target 3196 mutations (46\% of all pathogenic T > C variants), and A > G editors - 6900 mutations (34\% of all pathogenic G > A variants).

Conclusions: Protein engineering helps to develop new enzymes with a narrower window of base editors as well as using new Cas9 enzymes with different PAM sequences. But, even now the list of mutations which can be targeted with currently available systems is huge enough to choose and develop new targeted therapies.
\end{abstract}

Keywords: Base editor CRISPR/Cas9, ABE, APOBEC, PmCDA1, Pathogenic variants, Hereditary diseases

\section{Background}

There are currently over 6000 monogenic diseases according to OMIM [1]. Different DNA alterations may cause a disease, however the main reason of monogenic diseases is a pathogenic single nucleotide variant (SNV). SNVs account for approximately $90 \%$ of all records in

\footnotetext{
* Correspondence: alexandervlavrov@gmail.com

${ }^{1}$ Research Center for Medical Genetics, Moscow, Russia

Full list of author information is available at the end of the article
}

ClinVar [2] database (Fig. 1a), 23\% of which are pathogenic or likely pathogenic (Fig. 1b). Modern molecular genetic techniques, early diagnostics and advanced symptomatic and pathogenic treatment for many hereditary diseases are now available. Despite significant advancement in treating orphan diseases true cure is possible only by direct correction of mutated genes. Genome editing is thought to be the main breakthrough in treating monogenic diseases. The CRISPR/Cas9 system is one of the most popular tools to make changes in

(c) The Author(s). 2020 Open Access This article is licensed under a Creative Commons Attribution 4.0 International License, which permits use, sharing, adaptation, distribution and reproduction in any medium or format, as long as you give appropriate credit to the original author(s) and the source, provide a link to the Creative Commons licence, and indicate if changes were made. The images or other third party material in this article are included in the article's Creative Commons licence, unless indicated otherwise in a credit line to the material. If material is not included in the article's Creative Commons licence and your intended use is not permitted by statutory regulation or exceeds the permitted use, you will need to obtain permission directly from the copyright holder. To view a copy of this licence, visit http://creativecommons.org/licenses/by/4.0/ The Creative Commons Public Domain Dedication waiver (http://creativecommons.org/publicdomain/zero/1.0/) applies to the data made available in this article, unless otherwise stated in a credit line to the data. 

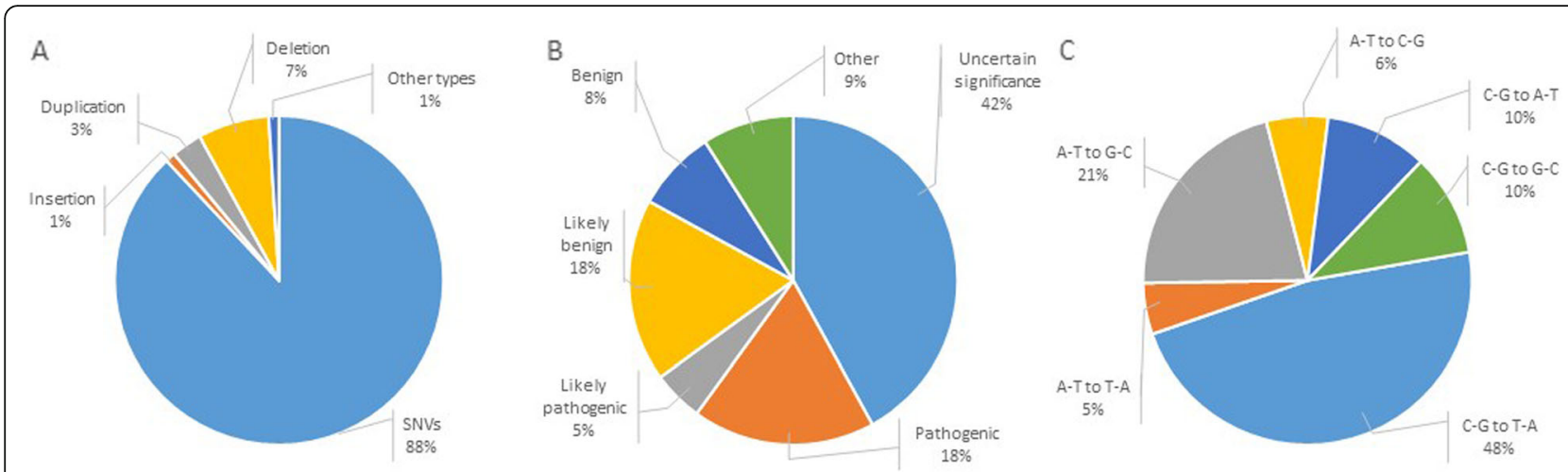

Fig. 1 ClinVar database analysis. a Types of mutations. Almost 90\% of them are single nucleotide variants, $\mathbf{b}$ Clinical significance of the mutations. Effects of more than 40\% variants registered in ClinVar are unknown, c Types of SNVs in humans leading to monogenic disorders

genome. It's based on inducing targeted single- or double-stranded break (DSB) in DNA which is then repaired by either non-homologous end joining (NHEJ) or homology directed repair (HDR). Both approaches are used for the development of new genome editing therapeutic approaches - HDR is used to correct targeted mutations while NHEJ can be used to universally skip exons with any pathogenic mutations [3]. However all developed methods have very low efficiency with high level of unwanted events mainly due to the DSB. Moreover it was reported that DSB may be the reason of large deletions and rearrangements [4]. NHEJ is the dominating DNA repair mechanism, but it's not precise and small insertions and deletions at the place of DSB are typical. Even in those cases when HDR successfully occurs the majority of DSBs are repaired by NHEJ.

New methods [5] can solve this problem by direct correction of individual nucleotides without inducing DSB repaired by NHEJ. CRISPR-Cas9-based single nucleotide editors developed recently may help to overcome the main obstacle in precise correction of SNVs. Their main characteristic is the direct change of the targeted nucleotide without inducing DNA breakes.

There are two major types of base editors (BEs). Earlier developed $\mathrm{C}->\mathrm{T}$ editors are built of CRISPRnuclease fused to cytidine deaminase [6]. Cas9/Cpf1 together with small guide RNA (sgRNA) target the construct to a specific DNA locus and cytidine deaminase converts $C$ to $T$. Later developed $A->G$ editors use adenine deaminase. Consequently both systems depend greatly on the properties of the CRISPR protein. Cas9 has a major PAM sequence NGG placed at the 3'end of the targeted locus. Cpf1 uses PAM at the $5^{\prime}$-end of the sgRNA. We use numerating of the nucleotides in this work starting from the PAM: -1 , $-2,-3 \ldots$ for Cas9 and $+1,+2,+3$ for Cpf1. Both systems can typically edit nucleotides in the range of 4-11 nucleotides (-17 - - 10 for Cas9) (Fig. 2). The width and position of the editing window depend on the properties of the deaminase and the linker between the deaminase and programmable nuclease. There are engineered nucleases with different PAMs which enlarges the number of potentially targetable DNA sequences. BEs don't need double-stranded DNA breaks because the can successfully work with nicks of the single DNA strand. This fact is very important for the development of safe DNA-editing systems with low risk of off-target events.

Here we describe all known BEs. We also performed analysis to find all possible pathogenic variants which can be efficiently targeted by any of the described systems and present them for further selection and development of targeted therapies.

\section{Methods}

ClinVar database (GRCh37_clinvar_20171203) was used to search and select mutations available for current single-base editing systems. We included only pathogenic and likely pathogenic variants for further analysis. Genome assembly hg19 was used as a reference.

Generally in order to target the specific mutation the Cas9-based system needs a PAM sequence. For every potentially editable mutation the PAM sequence should be in the interval dependent on the sgRNA length and

$$
\begin{aligned}
& \begin{array}{lllllllllllllllllllllll}
-20 & -19 & -18 & -17 & -16 & -15 & -14 & -13 & -12 & -11 & -10 & -9 & -8 & -7 & -6 & -5 & -4 & -3 & -2 & -1 & \text { Cas } 9 \text { PAM }
\end{array} \\
& \begin{array}{|llll|llllllll|lllllllllllll|}
\hline 5^{\prime}- & 1 & 2 & 3 & 4 & 5 & 6 & 7 & 8 & 9 & 10 & 11 & 12 & 13 & 14 & 15 & 16 & 17 & 18 & 19 & 20 & 21 & 22 & 23 & -3^{\prime} \\
\hline
\end{array} \\
& \begin{array}{lllllllllllllllllllll}
\text { Cpf1 PAM } & 1 & 2 & 3 & 4 & 5 & 6 & 7 & 8 & 9 & 10 & 11 & 12 & 13 & 14 & 15 & 16 & 17 & 18 & 19 & 20
\end{array}
\end{aligned}
$$

Fig. 2 Scheme of the targeted locus with numeration of the nucleotides depending on the Cas9 or Cpf1 used in the base editor 
width of the editing window of the specific BE. So the PAM sequence was searched in the window with coordinates [length sgRNA $-\mathrm{Y}$; length sgRNA $_{\text {s }}-\mathrm{X}+$ length $_{\text {PAM }}$ ] starting from mutation location (Fig. 3, a). Where length $_{\text {sgRNA }}$ is typically 20 for most of the systems, length $\mathrm{PAM}_{\mathrm{A}}$ is typically 3 and $\mathrm{X}$ and $\mathrm{Y}$ are the coordinates of the editing window for the particular $\mathrm{BE}$ if to count nucleotides from the $5^{\prime}$ end of the sgRNA. These calculations allowed to find the PAM in such a distance from the mutations that if and when $\mathrm{BE}$ would be applied the mutation will be found in the editable window.

If a PAM was found, we analyzed the editing window to find sequences with only one nucleotide (mutated) which can be edited without risk of changing neighboring nucleotides (Fig. 3, b). Detailed characteristics of the analyzed BEs are presented in the Table 1.

The code of the script to search the database and to analyze the sequences was written in $\mathrm{R}$ and is available in the Additional file 2.

\section{Results}

Editing systems are able to convert $\mathrm{G}(\mathrm{C})>\mathrm{A}(\mathrm{T})$ and $A(T)>G(C)$, which allows in theory to correct $68 \%$ of all mutations registered in ClinVar $(\mathrm{A}(\mathrm{T})>\mathrm{G}(\mathrm{C})-21 \%$ and $\mathrm{G}(\mathrm{C})>\mathrm{A}(\mathrm{T})-47 \%$ respectively) (Fig. 1,c). We selected only pathogenic and likely pathogenic mutations $-21 \%$ of all ClinVar records. Therefore, the total number of analyzed mutations was 27,310 .

We developed the $\mathrm{R}$ script to analyze 21 editing system currently reported in 9 publications. Every system has different working characteristics such as the editing window and PAM sequence which are summarized in the Table 1. C > T BEs have a lot of PAMs with the most popular NGG, and editing window is in the range of 20 to -5 . For $\mathrm{G}>\mathrm{A}$ mutations there are 2 systems with NGG/NGV/GAT PAMs and typical window from -17 to -12 .

Firstly, we searched for available PAMs near the target mutation (Fig. 2,a). Exact area of searching depends on the length of the editing window and length of the sgRNA. It was possible to find several PAMs in the designated area, which were analyzed individually. For all C $>$ T BEs, we found 6415 potential targets which constitutes $93 \%$ of all $\mathrm{T}>\mathrm{C}$ pathogenic mutations. ABE systems can edit 13,683 mutations $(67 \%$ of $\mathrm{G}>\mathrm{A}$ pathogenic mutations).

Then we analyzed editing windows around selected mutations to check for the presence of other $C(G)$ or $\mathrm{A}(\mathrm{T})$ nucleotides which could be nonspecifically edited together with targeted mutations. We selected only those mutations, which have no other targets near them (Table 2). As a result, for $\mathrm{C}>\mathrm{T}$ systems we select 3196 variants, it is approximately $46 \%$ of all pathogenic mutations, and 6900 mutations (34\% of all pathogenic) for A $>$ G systems.

The first successful single-base editor was presented in 2016 by A. Komor with colleagues [6]. The editor consists of nuclease-deficient Cas9 fused with APOBEC1 cytidine deaminase. Cas9 with sgRNA targets the complex to DNA. Deaminase converts any cytosine into uracil in the range of 8 nucleotides from -18 to -11 of the targeted sequence from PAM with the overall frequency of $37 \%$. Uracil is later repaired to thymine. The width and exact position of the window depends on the protein structure and linker length. Uracil glycosylase inhibitor was introduced to the complex to inhibit U-to-C back conversion.

\section{a. Determining the window to search the PAM}

\begin{tabular}{|l|l|l|l|}
\hline \multicolumn{1}{|l|}{ lengthsgR $\mathrm{A}-\mathrm{Y}$} & \multicolumn{1}{l|}{ PAM window } & \\
\hline
\end{tabular}

\section{b. Analyzing the editing window for the absence of other $\mathrm{C}$}

editing window $\mathrm{Y}$

NNNN C NNN

\section{PAM}

Fig. 3 Scheme of searching for potential targets for base editors. First the script searches for PAM near (yellow) the mutation based on the characteristics of the individual editor: PAM sequence and the editing window, in which the targeted nucleotide should fit (a). If the PAM is found in the necessary area, the script fixes its coordinates (green) and analyses the editing window (orange) to select only the window without other cytosine (or adenine) residues to reduce the risk of unwanted editing close to zero (b). a, search for PAM sequences around the mutation; $X$ - beginning of the editing window, $Y$ - end of the editing window. $b$, Analysis of the DNA sequence in the editing window around the selected mutation 
Table 1 Main characteristics of base editing systems

\begin{tabular}{|c|c|c|c|c|c|c|c|}
\hline Name & $\begin{array}{l}\text { Cas } \\
\text { protein }\end{array}$ & Deaminase & PAM & $\begin{array}{l}\text { Editing } \\
\text { window }\end{array}$ & $\begin{array}{l}\text { Editing } \\
\text { Efficiency }\end{array}$ & $\begin{array}{l}\text { Reference: Pubmed ID (Author, } \\
\text { Year) }\end{array}$ & $\begin{array}{l}\text { Edited mutated } \\
\text { nucleotides }\end{array}$ \\
\hline APOBEC & dCas9 & APOBEC 1 & NGG & -18 to -11 & $15-75 \%$ & 27,096,365 (Komor, 2016) [6] & \\
\hline SaBE3 & SaCas9n & APOBEC 1 & NNGRRT & -15 to -9 & $5-65 \%$ & & $\mathrm{~T}>\mathrm{C}$ \\
\hline SaKKH-BE3 & dCas9 & APOBEC 1 & NNNRRT & -17 to -9 & $10-65 \%$ & 28,191,901 (Kim, 2017) [7] & \\
\hline EQR-Cas9 & dCas9 & APOBEC 1 & NGAG & -17 to -10 & $10-40 \%$ & & \\
\hline VRER-Cas9 & dCas9 & APOBEC 1 & NGCG & -19 to -10 & $10-35 \%$ & & \\
\hline VQR-Cas9 & dCas9 & APOBEC 1 & NGAN & -17 to -10 & $10-60 \%$ & & \\
\hline YE1-VQR-Cas9 & dCas9 & APOBEC 1 & NGAN & -16 to -15 & $10-30 \%$ & & \\
\hline A-BE3 & dCas9 & APOBEC 1 & NGG & -17 to -12 & $20-50 \%$ & & \\
\hline Y-BE3 & dCas9 & APOBEC 1 & NGG & -17 to -13 & $10-30 \%$ & & \\
\hline FE-BE3 & dCas9 & APOBEC 1 & NGG & -16 to -14 & $10-40 \%$ & & \\
\hline YEE-BE3 & dCas9 & APOBEC 1 & NGG & -16 to -15 & $5-35 \%$ & & \\
\hline PmCDA1 & nCas9 & PmCDA1 & NGG & -20 to -16 & $6-96 \%$ & 27,492,474 (Nishida, 2016) [8] & \\
\hline BE-PLUS & nCas9 & APOBEC 1 & NGG & -16 to -5 & $10-30 \%$ & 29,875,396 (Jiang, 2018) [9] & \\
\hline xCas9-BE3 & xCas9 & APOBEC 1 & $\begin{array}{l}\text { NGN, } \\
\text { GAW }\end{array}$ & -17 to -13 & $10-24 \%$ & $29,512,652(\mathrm{Hu}, 2018)[10]$ & \\
\hline dCpf1-eBE & dCpf1 & APOBEC 1 & TTV & 8 to 13 & $15-30 \%$ & $29,553,573$ & \\
\hline dCpf1-eBE-YE & dCpf1 & APOBEC 1 & TTV & 10 to 12 & $2-28 \%$ & $(\mathrm{LI}, 2018)[1 \mathrm{I}]$ & \\
\hline $\begin{array}{l}\text { APOBEC3A- } \\
\text { Cas9 }\end{array}$ & nCas9 & APOBEC $3 \mathrm{~A}$ & NGG & -16 to -12 & $16-48 \%$ & 30,059,493 (Gehrke, 2018) [12] & \\
\hline $\begin{array}{l}\text { EA3A- } \\
\text { BE3(VRQR) }\end{array}$ & xCas9 & APOBEC $3 \mathrm{~A}$ & NGAN & -17 to -10 & $15-63 \%$ & & \\
\hline $\begin{array}{l}\text { EA3A- } \\
\text { BE3(xCas9) }\end{array}$ & xCas9 & APOBEC $3 \mathrm{~A}$ & NGG, NGT & -17 to -13 & $17-35 \%$ & & \\
\hline BE-PAPAPAP & nCas9 & APOBEC 1 & NGG & -16 to -15 & $24 \%$ & 30,683,865 (Tan, 2019) [13] & \\
\hline CCDA1-BE3 & nCas9 & CDA1 & NGG & -19 to -16 & $50 \%$ & & \\
\hline xCas9-ABE & xCas9 & TadA & NGV, GAT & -17 to -13 & $16-69 \%$ & $29,512,652(\mathrm{Hu}, 2018)[10]$ & $G>A$ \\
\hline TadA & dCas9 & TadA & NGG & -17 to -12 & $25-75 \%$ & 29,160,308 (Gaudelli, 2017) [14] & \\
\hline
\end{tabular}

And finally the authors partially restored nuclease activity to cut the strand complementary to the converted nucleotide. This editor was called third-generation base editor BE3. Later the same authors managed to develop additional systems with different editing windows and PAM sequences by changing deaminase linker length and Cas9 enzyme [7]. They succeeded in reducing window by different mutations: -17 to -12 for A-BE3(R126A), -17 to -13 for Y-BE3(W90Y), -16 to -14 for FE$\mathrm{BE} 3(\mathrm{~W} 90 \mathrm{~F}+\mathrm{R} 126 \mathrm{E})$ and -16 to -15 for YEEBE3(W90Y + R126E + R132E). Also, authors analyzed new Cas9 variants with altered PAMs: NGAN (VQR-Cas9) with -17 to -10 window and YE1-VQR-BE3 with -16 to -15 window, NGAG (EQR-Cas9) with -17 to -10 window, NGCG (VRER-Cas9) with -19 to -10 window. In addition, they use Cas9 homolog from Staphylococcus aureus (SaCas9) with PAM NNGRRT ( -15 to -9 window) and an engineered SaCas9 variant containing three mutations (SaKKH-Cas9) with PAM NNNRRT ( -15 to 9 window).
K. Nishida with colleagues presented a very similar editor based on another enzyme - activation-induced cytidine deaminase (PmCDA1) and nCas9 (D10A) [8]. The main difference was the editing window from -20 to -16 nucleotide of the targeted sequence. System demonstrated approximately $60 \%$ editing frequency in mammalian cells, with off-target mutations in lower than $1.5 \%$. We found that nCas9(D10A)-PmCDA can target $2544 \mathrm{~A}(\mathrm{~T})>\mathrm{G}(\mathrm{C})$ mutations and 566 of them may be corrected without affecting nearby nucleotides.

$\mathrm{W}$. Jiang with his team made a system with the longest editing window from -16 to -5 [9]. In $2018 \mathrm{~J} \mathrm{Hu}$ et al. described modified Cas protein (xCas9) with increased number of PAMs: NG, GAA, and GAT [10].

Not only PAM sequence but also its position relative to the targeted mutations limits the usage of BEs, especially in the AT-rich regions, which are difficult to find PAMs typical for Cas9-based systems. Cpf1 has a different PAM sequence - TTTV which is also recognized upstream from the targeted sequence 
Table 2 Numbers of mutations targetable by different base editors

\begin{tabular}{|c|c|c|}
\hline C > T Systems & Number of mutations & Number of potential sgRNAs \\
\hline A-BE3 & 538 & 655 \\
\hline APOBEC & 397 & 502 \\
\hline APOBEC3A-Cas9 & 115 & 403 \\
\hline BE_PLUS & 181 & 229 \\
\hline EQR_Cas9 & 144 & 152 \\
\hline FE-BE3 & 714 & 822 \\
\hline PmCDA1 & 566 & 687 \\
\hline SaCas9 & 122 & 122 \\
\hline SaKKH_BE3 & 424 & 452 \\
\hline VQR_Cas9 & 485 & 530 \\
\hline VQR_Cas9_eA3A & 766 & 766 \\
\hline VRER_Cas9 & 28 & 29 \\
\hline Y-BE3 & 599 & 722 \\
\hline YEE-BE3 & 720 & 791 \\
\hline dCpf1-eBE & 136 & 136 \\
\hline dCpf1-eBE-YE & 164 & 164 \\
\hline eA3A_xCas9 & 164 & 634 \\
\hline xCas9_BE3 & 2098 & 3001 \\
\hline Total number of mutations & 3196 & \\
\hline \multicolumn{3}{|l|}{ A > G Systems } \\
\hline TadA & 2568 & 3235 \\
\hline xCas9_ABE & 6829 & 9638 \\
\hline Total number of mutations & 6900 & \\
\hline
\end{tabular}

Some of the mutations can be targeted using more than one PAM, that's why the number of potential sgRNAs can be bigger than the number of mutations

unlike NGG which goes immediately after targeted DNA. Cpf1 fusion with APOBEC1 allows targeting AT-rich sequences [11]. There are 2 systems with different editing windows: $\mathrm{dCpf} 1-\mathrm{eBE}$ from 8 to 13 and dCpf1-eBE-YE from 10 to 12.

J. Gehrke and his team tried to develop more precise BE3-based systems depending on the nucleotides neighboring the targeted mutation with $\mathrm{TCR}>\mathrm{TCY}>\mathrm{VCN}$ hierarchy [12].

Most of the pathogenic mutations are $\mathrm{G}(\mathrm{C})>\mathrm{A}(\mathrm{T})$ substitutions (47\%) (Fig. 1, C). That is why adenine base editor would be of great practical importance allowing correction of almost half of all mutations. However there are no natural enzymes able to convert $A(T)$ to $G(C)$. By direct genetic and protein engineering adenine base editor (ABE) was developed by Gaudelli NM et al. [14]. $\mathrm{ABE}$ consists of adenine deaminase TadA and Cas9 protein (ABE7.10). Substitution of adenine to guanine occurs in a window from -17 to -12 nucleotides of the targeted sequence with a probability of $60 \%$. ABE7.10 base editor can target $7044 \mathrm{G}(\mathrm{C})>\mathrm{A}(\mathrm{T})$ mutations in the -17 / -12 nucleotide window. Over $2 / 3$ of them (2568) can be specifically targeted in the regions without other
$\mathrm{A}(\mathrm{T})$. With modification of Cas9 and availability of additional PAMs [9] the system managed to target almost 3 times more mutations - 6829 .

The full list of all targetable mutations is available in the Additional file 1.

\section{Discussion}

Single base editors (BE) are very promising genetic tools for safe targeted correction of single nucleotide variants. They reduce the risk of indels aroused during repairing double stranded breaks. However base editors have wide editing windows and this fact which limits their potential use in editing targeted single nucleotides. Usually each nucleotide is repeated in DNA sequence in the range of 8-10 nucleotides which is the typical window width of base editors. Though there is a significant progress in the development of new BE with narrow editing windows [11] unfortunately, none of the BEs is ideally specific. Even recently developed highly specific editors claimed by the authors to edit 1-2 nucleotides at some tested loci still have a window of several nucleotides edited at very low frequency [13]. It means that if there are several targets in the window, the enzyme can edit all of 
Table 3 Possible base editing systems to correct pathogenic variant NM_001005463.2:c.196A > G responsible for ataxia with delayed development

\begin{tabular}{lll}
\hline Base editor & Editing window & Protospacer genome sequence \\
\hline A-BE3 & attgga & gaaatTggatttccggaggttgg \\
Y-BE3 & attgg & gaatTggatttccggaggttgg \\
FE-BE3 & ttg & gaaatTggatttccggaggttgg \\
YEE-BE3 & tt & gaaatTggatttccggaggttgg \\
VQR_Cas9 & ttggattt & aaatTggatttccggaggttggaa \\
YE1-VQR-Cas9 & tg & aaatTggatttccggaggttggaa \\
APOBEC & aagaaatt & ggaagaaatTggatttccggagg \\
APOBEC & aatTggat & gaaatTggatttccggaggttgg \\
BE_PLUS & ggaagaaatTgga & agtggaagaaatTggatttccgg \\
BE_PLUS & agaaatTggattt & ggaagaaatTggatttccggagg \\
XCas9_BE3 & attgg & gaaatTggatttccggaggttgg \\
xCas9_BE3 & ttgga & aaatTggatttccggaggttgga \\
XCas9_BE3 & tggat & aatTggatttccggaggttggaa \\
APOBEC3A-Cas9 & ttgga & gaaatTggatttccggaggttgg \\
eA3A_XCas9 & attgg & gaaatTggatttccggaggttgg \\
SaKKH_BE3 & gaagaaatt & gtggaagaaatTggatttccggaggt \\
PmCDA1 & ttgga & tTggatttccggaggttggaagg \\
\hline
\end{tabular}

them, but not only the desired target. It's reasonable to select the most safe targets for possible genome editing with $\mathrm{BE}$ especially for the development of treatment in vivo. Therefore we analyzed editing windows around selected mutations to select only those which can be edited absolutely safely.

We demonstrated that about $37 \%$ of all pathogenic and likely pathogenic single nucleotide variants can be safely edited without chances to convert neighbor nucleotides. These mutations are found in 2364 genes and are responsible for the development of 4000 diseases or syndromes (based on MedGen https://www. ncbi.nlm.nih.gov/medgen/). It's interesting to note, that 779 mutations can be edited by more than 3 analyzed BEs, which opens great potential for optimizing editing protocols.

For example one pathogenic variant NM_001005463.2: c.196A > G described in ataxia with delayed development (OMIM 617330) can be targeted by 13 different systems with 17 different sgRNAs (Table 3).

The non-mutated $\mathrm{T}$ in the genome is highlighted in bold capital letter. Since the real $B E$ converts $C>U>T$, all $\mathrm{A}>\mathrm{G}$ mutations were also converted to complementary sequences and algorithm was applied to the complementary sequence (containing $\mathrm{C}$ as a mutation but not $G$ ) if necessary. That is why the table contains only "T"s as reference nucleotides. Despite big difference in the editing length none of the windows contains Cytosine, which could be unintentionally edited together with $\mathrm{T}>\mathrm{C}(\mathrm{A}>\mathrm{G})$.

\section{Conclusions}

CRISPR/Cas9 base editors allow to precisely target $46 \%$ of all $\mathrm{T}>\mathrm{C}$ pathogenic mutations and $34 \%$ of all $\mathrm{G}>\mathrm{A}$ pathogenic mutations. Protein engineering helps to develop new enzymes with even narrower window of editing which makes the editors more precise. Newly engineered Cas9 enzymes recognize various PAM sequences. Additionally the linker length between Cas9 and deaminase may help to shift the editing window to further widen the capabilities of base editors. However, even now the list of mutations which can be targeted with currently available systems is huge and allows to choose and to develop new targeted genome editing therapies.

\section{Supplementary information}

Supplementary information accompanies this paper at https://doi.org/10. 1186/s12920-020-00735-8.

Additional file 1. Table with the full list of all targetable pathogenic variants.

Additional file 2. Code of the script.

\section{Acknowledgements}

Not applicable.

\section{About this supplement}

This article has been published as part of BMC Medical Genomics Volume 13 Supplement 8, 2020: Selected Topics in "Systems Biology and Bioinformatics" - 2019: medical genomics. The full contents of the supplement are available online at https://bmcmedgenomics.biomedcentral.com/articles/supplements/ volume-13-supplement-8. 


\section{Authors' contributions}

AVL generated and developed the concept and general idea of the study, discussed and analyzed the results, wrote the manuscript. GGV developed the code of the script, analyzed the data, drafted the manuscript. MYS developed the idea of the study, algorithm of the data collection and analysis, discussed the results, drafted the manuscript. The authors read and approved the final manuscript.

\section{Funding}

The research was carried out within the state assignment of Ministry of Science and Higher Education of the Russian Federation, which covered the research and writing of the article. Publication costs are funded personally.

\section{Availability of data and materials}

All data generated or analyzed during this study are included in this published article and its supplementary information files.

\section{Ethics approval and consent to participate}

Not applicable.

\section{Consent for publication}

Not applicable.

\section{Competing interests}

The authors declare that they have no competing interests.

\section{Author details}

${ }^{1}$ Research Center for Medical Genetics, Moscow, Russia. ${ }^{2}$ Moscow Institute of Physics and Technology, Dolgoprudny, Russia. ${ }^{3}$ School of Biomedicine, Far Eastern Federal University, Vladivostok, Russia.

Received: 13 May 2020 Accepted: 1 June 2020

Published: 18 September 2020

\section{References}

1. Online Mendelian Inheritance in Man, OMIM*: McKusick-Nathans Institute of Genetic Medicine, Johns Hopkins University (Baltimore, MD); 2019. https://omim.org/ Accessed 15 Jan 2019.

2. Landrum MJ, Lee JM, Benson M, Brown GR, Chao C, Chitipiralla S, et al. ClinVar: improving access to variant interpretations and supporting evidence. Nucleic Acids Res. 2018;46:1062-7.

3. Long C, Li H, Tiburcy M, Rodriguez-Caycedo C, Kyrychenko V, Zhou H, Zhang $Y$, et al. Correction of diverse muscular dystrophy mutations in human engineered heart muscle by single-site genome editing. Sci Adv. 2018;4(1):eaap9004.

4. Kosicki M, Tomberg K, Bradley A. Repair of double-strand breaks induced by CRISPR-Cas9 leads to large deletions and complex rearrangements. Nat Biotechnol. 2018;36:765-71.

5. Zhang C, Quan R, Wang J. Development and application of CRISPR/Cas9 technologies in genomic editing. Hum Mol Genet. 2018;27:79-88.

6. Komor AC, Kim YB, Packer MS, Zuris JA, Liu DR. Programmable editing of a target base in genomic DNA without double-stranded DNA cleavage. Nature. 2016;533:420-4.

7. Kim YB, Komor AC, Levy JM, Packer MS, Zhao KT, Liu DR. Increasing the genome-targeting scope and precision of base editing with engineered Cas9-cytidine deaminase fusions. Nat Biotechnol. 2017;35:371-6.

8. Nishida K, Arazoe T, Yachie N, Banno S, Kakimoto M, Tabata M, et al. Targeted nucleotide editing using hybrid prokaryotic and vertebrate adaptive immune systems. Science. 2016;353:aaf8729.

9. Jiang W, Feng $S$, Huang $S$, Yu W, Li G, Yang G, et al. BE-PLUS: a new base editing tool with broadened editing window and enhanced fidelity. Cell Res. 2018;28(8):855-61.

10. Hu JH, Miller SM, Geurts MH, Tang W, Chen L, Sun N, et al. Evolved Cas9 variants with broad PAM compatibility and high DNA specificity. Nature. 2018;556(7699):57-63.

11. Li X, Wang Y, Liu Y, Yang B, Wang $X$, Wei J, et al. Base editing with a Cpf1-cytidine deaminase fusion. Nat Biotechnol. 2018;7:324-7.

12. Gehrke JM, Cervantes O, Clement MK, Wu Y, Zeng J, Bauer DE, et al. An APOBEC3A-Cas9 base editor with minimized bystander and off-target activities. Nat Biotechnol. 2018;36(10):977-82.
13. Tan J, Zhang F, Karcher D, Bock R. Engineering of high-precision base editors for site-specific single nucleotide replacement. Nat Commun. 2019; 10:439.

14. Gaudelli NM, Komor AC, Rees HA, Packer MS, Badran AH, Bryson DI, et al. Programmable base editing of a.T to $\mathrm{G} \cdot \mathrm{C}$ in genomic DNA without DNA cleavage. Nature. 2017;551:464-71.

\section{Publisher's Note}

Springer Nature remains neutral with regard to jurisdictional claims in published maps and institutional affiliations.

\section{Ready to submit your research? Choose BMC and benefit from:}

- fast, convenient online submission

- thorough peer review by experienced researchers in your field

- rapid publication on acceptance

- support for research data, including large and complex data types

- gold Open Access which fosters wider collaboration and increased citations

- maximum visibility for your research: over $100 \mathrm{M}$ website views per year

At BMC, research is always in progress.

Learn more biomedcentral.com/submissions 